\title{
Temporalité de l'écriture et génétique textuelle : Vers un autre métalangage?
}

\author{
Doquet, Claire et Leblay, Christophe \\ Université de Paris Sorbonne Nouvelle et Université de Turku (Finlande) \\ claire.doquet@univ-paris3.fr et christophe.leblay@utu.fi
}

La génétique textuelle, fondée dans les années 1970 en vue d'explorer des manuscrits d'écrivains, est confrontée aujourd'hui à un matériaux d'un type nouveau: les données recueillies informatiquement qui retracent, le plus souvent, sous la forme de films, le processus de l'écriture. Que l'outil scriptural soit un ordinateur ou un stylo, des logiciels existent qui permettent aux observateurs d'accéder aux traces, ordonnées spatialement et chronologiquement, de la constitution d'un texte. Depuis la création, en France, du logiciel Genèse du texte produit par l'Association Française pour la Lecture en 1993 (swww.lecture.org) et premier logiciel de traitement de texte, semble-t-il, a avoir enregistré et restituée la procédure d'écriture des textes, et à en avoir proposé un outillage analytique, un certain nombre d'outils se sont succédés et les dernières années ont vu naître divers stylos et logiciels de traitement de texte permettant d'enregistrer l'écriture. Cette mise à disposition des traces de la temporalité scripturale paraît résoudre un des problèmes cruciaux de la génétique textuelle : la reconstitution de la procédure de constitution du texte est, lors de l'étude de manuscrits, une étape fondamentale de la recherche. Cette temporalité montrée pose à la génétique des questions que les manuscrits laissaient dans l'ombre, en particulier celle du rythme de l'écriture et de ses incidences sur le texte et l'activité du scripteur. Elle pose également des questions neuves à la linguistique dans son effort de spécifier l'énonciation écrite : quel est le statut des pauses dans cette énonciation singulière où la chronologie des événements d'énonciation reflète rarement l'ordre des énoncés dans le texte final ? D'un point de vue énonciatif, comment caractériser ce qui se joue pendant les pauses? Quel statut donner aux opérations intervenant au sein du déjà écrit, quel peut être leur lien avec la continuation du texte?

Le matériau fourni par les outils d'enregistrement de l'écriture est souvent appelé "écriture en temps réel », expression forgée sur le modèle de l'anglais on line et que l'on trouve dans les travaux les plus anciens du domaine (Foulin, 1988; Foulin, Chanquoy \& Fayol, 1989; Chesnet, Guillabert \& Esperet, 1994 ; Doquet, 1995). Cette expression nous paraît approximative puisque ce que les logiciels permettent d'observer n'est pas l'écriture au moment où elle se produit (on line) mais une reconstitution des événements qui l'ont composée. Aussi, nous préférons parler d'écriture enregistrée, suivant en cela les propositions de Leblay et Caporossi (2014, sous presse, p. 12). Cette appellation est due également à un effort d'uniformisation entre deux langues, puisque la langue anglaise propose keystroke logging en parallèle au terme de Real-time operating system (RTOS), qui décrit un système d'exploitation en temps réel, et non une méthode de description du processus d'écriture.

Après une description détaillée du nouveau matériau que constitue l'écriture enregistrée, nous montrerons quels problèmes de traitement elle pose par rapport à l'outillage existant, celui de la génétique textuelle. Puis, nous montrerons les écueils et les avantages des différentes transcriptions de l'écriture enregistrée qui ont été proposées depuis 20 ans et nous tenterons de dégager les tendances de cette évolution. 


\section{L'écriture enregistrée : un nouveau matériau pour la génétique textuelle}

\subsection{Description du matériau}

Enregistrée au moment de son effectuation par le scripteur, l'écriture est restituée sous la forme d'un film, avec l'ensemble de ses caractéristiques spatio-temporelles. L'écriture enregistrée apporte donc comme données des éléments que la génétique textuelle cherche, depuis sa naissance, à reconstituer. Louis Hay écrivait à propos de la génétique textuelle $(2009,24)$ que cette dernière « n'a pas pour autant fait revivre la genèse dans sa réalité temporelle ». Pour Lebrave, on peut

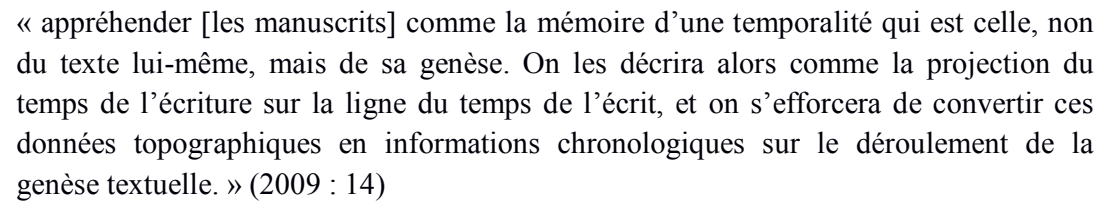

La distinction entre les termes de manuscrit et de brouillon est ainsi posée pour Bellemin-Noël (1982, pp. 161-162) : le manuscrit désigne, « en toute rigueur, des feuilles de papier couvertes de signes tracés par la main de l'écrivain ». En revanche, le terme de brouillon sert à désigner «la matérialisation d'un discours inachevé, prospectif, parfois mis au rebut, le plus souvent transformé au fil d'une pratique d'élaboration», en permettant de distinguer le pluriel, les brouillons, conçus comme ensemble de «tout ce qui a servi à la composition d'un ouvrage, [de] ce qui n'a jamais eu le statut du publiable » du singulier, le brouillon, compris comme «le premier jet et ses métamorphoses (ajouts, corrections, ratures et substitutions) jusqu'à l'état final de la première publication ». C'est bien le brouillon que construit la génétique. La temporalité de l'écriture est mise en avant par les linguistes qui s'attachent à décrire l'activité scripturale : pour Fabre-Cols, l'écriture est «fondamentalement dans le temps » $(2004,21)$; Boré (1996:135) insiste sur l'importance de la prise de conscience par des scripteurs scolaires du «tempo de la réécriture »; pour Ganascia, Fenoglio et Lebrave (2004) « toute œuvre est le résultat d'un processus de production qui, comme tel, est inscrit dans une temporalité ».

Le matériau dont nous traitons ici est de l'ordre du brouillon comme «premier jet et ses métamorphoses jusqu'à l'état final». Il intéresse évidemment la génétique textuelle mais aussi singulièrement la linguistique, pour les raisons annoncées en introduction : ce matériau, avec les informations nouvelles et stables qu'il donne sur les variables temporelles de l'écriture, permet de croiser les hypothèses formulées par les généticiens et les linguistes - voire, parfois, les linguistes généticiens - à propos de la nature et des modalités de l'énonciation écrite. Notre propos ici n'est pas de faire le point sur ces conséquences théoriques mais de mettre en évidence, à travers l'analyses des différences entre les manuscrits et l'écriture enregistrée, certains des déplacements qu'elles provoquent dans la délimitation et la catégorisation des faits, déplacements qui ont bien entendu des conséquences importantes sur les représentations de l'écriture et les principes et modalités d'étude.

\subsection{Saillances dans un manuscrit versus dans un film d'écriture}

La nature des éléments rendus visibles sur les manuscrits ont présidé à l'élaboration des catégories de la génétique textuelle : opérations (ajout, suppression, remplacement, déplacement), variantes de lecture et d'écriture (Grésillon 1994). Dans l'écriture enregistrée, les éléments les plus visibles ne sont pas les mêmes : 
Tableau 1. Saillances contrastées

\begin{tabular}{|l|l|}
\hline MANUSCRIT & FILM \\
\hline Ratures & Pauses \\
\hline Disposition des segments & Déplacement du curseur/stylo \\
\hline Unité de la page & Unité de la période d'écriture \\
\hline Mots écrits & Ecriture des mots \\
\hline
\end{tabular}

Ce constat conduit à rénover l'outillage conceptuel qui permet le traitement de ces données. Il faut se garder de confondre deux réalités souvent liées mais non assimilables : l'opposition entre écriture manuelle et écriture sur traitement de texte d'une part, l'opposition entre écriture enregistrée et manuscrits ou tapuscrits d'autre part. Certes, les corpus «papier» le plus souvent examinés sont manuscrits ; certes, les corpus enregistrés le plus souvent recueillis sont sur traitement de texte. Pour autant, il s'agit d'une régularité de distribution, pas d'un lien de causalité.

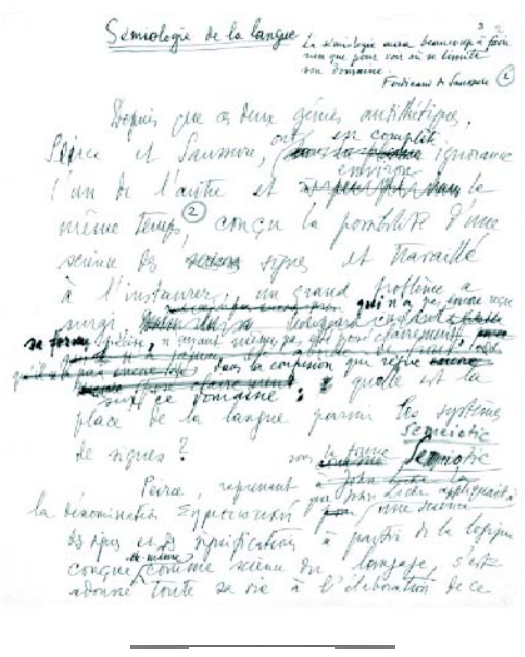

Graphique 1. Première page de «Sémiologie de la langue »

\section{Que montre, ou pas, cette page d'écriture réalisée sur papier?}

Tout d'abord, autant le manuscrit que le brouillon coexiste sur cette même page : il s'agit bien d'une feuille de papier couverte de signes tracés par la main de Benveniste tout autant qu'un premier jet et ses métamorphoses (ajouts, corrections, ratures et substitutions). De ces deux statuts, le second est celui qui intéresse la description linguistique, le premier intéressant principalement la codicologie. 
Ce manuscrit, devenu donc brouillon, nous montre l'importance des ratures : il ne viendrait à personne l'idée de mettre en doute les compétences scripturales que peut avoir un auteur comme Benveniste. Il est donc nécessaire de considérer les manuscrits de linguistes qui offrent une contribution indispensable pour la compréhension des phénomènes scripturaux (Fenoglio, 2012).

Néanmoins, ce brouillon ne peut pas nous montrer la chronologie des retours et le jeu des pauses. Il peut y avoir des hypothèses, certes, mais rien que des hypothèses. Combien de temps Benveniste a-t-il pris pour écrire et réécrire ces lignes ? Dans quel ordre a-t-il procédé ? Ou et combien de temps a-t-il pausé ?

Je ne vois pas un lieu ou un endroit précis comme élément essentiel si je pense à un cadre de vie idéal. La géographie 'extérieure' a une certaine importance, oui, mais c'est la géographie 'intérieure' qui crée les conditions de vie idéales. Par géographie intérieure j'entends ce qu'on pourrait appeler connaissance de soi-même et l'équilibre dans les émotions et dans les relations affectives. Il est impossible d'établir une liste des personnes qui feraient partie de ce cadre de vie idéale. En fait, la composition peut varier selon les situations. L'essentiel est que la relation avec les individus qui me sont proches à tel moment soit ouverte et satisfaisante pour moi ${ }^{\mathrm{p}}$ comme pour l'autre. Ces relations apportent alors un bien-être mental qui permet de vivre en principe (relativement) heureux, et si on rencontre des difficultés, de les surmonter.

Il faut toutefois admettre qu'un certain confort matériel contribue au sentiment de bonheur.

Graphique 2. Page imprimée d'une écriture numérique

\section{Que montre, ou pas, cette (page d') écriture réalisée sur écran?}

La désignation même de feuille de papier, de page est même remise en question, puisque ce qui est donné à voir est une simple mise en scène : il est bien plus facile de continuer de parler de page, unité visuelle commode, pour désigner ce qui est une saisie d'écran. Néanmoins, en référence à l'interrogation portant sur l'écriture sur papier, autant le statut de manuscrit que celui de brouillon semblent être neutralisés par un effet de mise au propre immédiat, représentatif d'un tapuscrit. A proprement parler, il n'y a plus de feuille de papier couverte de signes tracés par la main de son auteur, représentatif du manuscrit, et pas davantage un premier jet et ses métamorphoses (ajouts, corrections, ratures et substitutions), représentatif du brouillon. De ces deux statuts, le présent document ne semble en retenir donc aucun, contrairement au document précédent qui, lui, offrait, en couches superposées, les deux statuts. Cette différence majeure, dans la représentation du travail en production écrite, nous amène à nous interroger sur la légitimité de telle ou telle représentation : ne serait-il pas alors plus pertinent de continuer de travailler à partir d'un support qui permette de voir de suite les traces laissées, comme le font le papier et le crayon? Ce serait oublier deux arguments. Le premier est que le support papier ne donne que des traces de modifications spatiales, sans aucune possibilité de repérage temporel de ces traces. Le second argument concerne l'évolution des logiciels d'aide à l'écriture, tels le traitement de texte : le support numérique propose de plus en plus de solutions d'accès à ce qu'a été le déroulement temporel d'une écriture. Des fonctions, telles Time Machine, permettent de pouvoir revenir, à chaque instant, à une chaîne de versions antérieures. D'une manière générale, les traitements de texte ont intégré, systématiquement, la fonction Révision (écriture-réécriture). Cette évolution dans la manière de concevoir l'aide à l'écriture-réécriture reste déterminante pour l'avenir en plaçant au premier plan l'idée qu'il ne peut y avoir d'amélioration sans retour dans son écriture. Cette écriture, réalisée sur un écran, donne donc accès au temps de l'écriture, tout simplement parce qu'il existe 
un outillage pour prendre en compte la succession des versions produites au cours du processus d'écriture, ce que ne peut pas faire une page écrite sur un papier.

\section{L'outillage classique de la génétique et les problèmes qu'il pose pour analyser l'écriture enregistrée}

\subsection{Les 4 opérations "fondamentales" de l'écriture}

$\mathrm{Au}$ moment de son développement dans les années 1970, la génétique textuelle s'est employée, en particulier au sein de l'équipe Manuscrits et linguistique de l'Item $^{1}$, à forger des outils d'investigation applicables à toute écriture :

- tout manuscrit est analysable comme le résultat d'une suite de substitutions qui sont : des ajouts, des suppressions, des remplacements et des déplacements ;

- la notion de substitution n'est pas symétrique mais nécessairement orientée par la chronologie de l'écriture, déterminante pour l'explication du processus ;

- les catégories de substitutions ${ }^{2}$, que les manuscrits ne permettent d'envisager que comme des modifications apportées à une version initiale de l'écrit, sont déterminées à l'aide de leurs traces graphiques : ratures proprement dites et ajouts en marge, en interligne, etc. Ces traces permettent d'inférer qu'une substitution a été effectuée immédiatement après l'inscription du segment sur lequel elle porte, ou bien non immédiatement.

C'est en termes d'opérations que les choses sont décrites et ce choix correspond à une position épistémologique originale : envisager l'écriture comme un processus qui ne s'arrête pas aux états d'un écrit mais où est centrale l'activité du sujet énonciateur ; s'intéresser à ce que Louis Hay appelle « la troisième dimension du texte », «l'épaisseur de son devenir » :

«Le sort de l'œuvre se joue avec des élans et des épuisements, des bégaiements et des vides, des ruptures et des inachèvements qui nous déroutent. Le texte ne s'abolit pas dans cette profondeur de champ, il apparaît seulement comme un objet bien plus complexe que les modèles anciens d'une œuvre, bien plus aléatoire que ses modèles actuels. » (Hay, $2002: 58)$

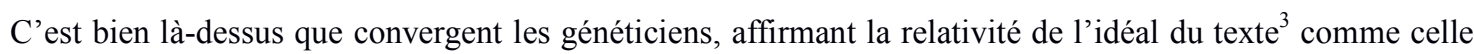
de sa clôture ${ }^{4}$, forts du principe que «ce qui a été écrit n'est pas plus prestigieux ni révélateur ni moins significatif d'avoir été barré ou biffé » (Bellemin-Noël, 1982, p. 163). Dès lors, c'est l'écriture en acte qui intéresse, avec son corrélat, le sujet. ${ }^{6}$ Cette écriture s'affirme comme le centre de l'intérêt des chercheurs, c'est pour la décrire qu'ils mettent au point le paradigme de la génétique textuelle. Et tout de suite, un paradoxe : sur les manuscrits, ce n'est pas le mouvement de l'écriture mais son résultat, même provisoire, qui est rendu visible. L'écriture est reconstituée à partir des instantanés que constituent les différents feuillets témoignant des états intermédiaires de l'écrit. Bien évidemment l'appréhension même de l'acte d'écrire est informée du matériau et des informations que les chercheurs sont capables d'y discerner : tout en affirmant son intérêt pour l'écriture en acte, la génétique travaillant sur des manuscrits s'appuie sur des objets figés. Voici quelques exemples des limites que posent les manuscrits à l'analyse scripturale. 


\subsection{L'ajout : une opération difficile à caractériser}

Le terme d'ajout est réservé, dans l'étude des manuscrits, à l'inscription d'éléments graphiques à l'entour du texte, entre les lignes, voire entre les mots, dans les espaces laissés blancs par le texte préalablement. L'étude génétique des manuscrits part d'un premier état de l'écrit et s'appuie sur les traces des opérations effectuées sur cet existant. Cette catégorisation est liée au matériau étudié, qui pose comme un être-là le texte central et offre entre autres accroches pour reconstituer la chronologie de l'écriture les interventions dans les à-côtés. Grâce au positionnement des énoncés, on détermine en premier lieu deux entités : le texte central, dont l'ébauche a vraisemblablement constitué le début de l'écriture, et les entours, les marges, qui viennent moduler ce texte.

Le traitement de texte offre un espace tout différent, sans rature, malléable à l'infini, mais contraignant aussi par la linéarité qu'il impose. Qui a observé, dans les manuscrits de Valéry, l'immixtion du dessin et du texte, a du mal à imaginer cet écrivain devant un ordinateur. Sur traitement de texte, sauf utilisations marginales, pas de dessin, pas de sortie de la ligne pré-tracée, pas de marge. Tout élément inscrit est fondu illico dans l'écrit existant, à moins de jeux typographiques bien plus difficilement réalisables au clavier qu'au stylo. Ceci signifie, du point de vue du scripteur, que toute modification s'intègre à l'énoncé comme si elle en avait toujours fait partie, étouffant ainsi le caractère historique de la constitution du texte qui fait place à l'impression d'un objet toujours propre, toujours neuf, non altéré par les aléas de son écriture. C'est l'anti-ajout, parce que l'anti-rature, si l'on ne considère comme des ajouts que ce qui est hors linéarité. Mais dans l'écriture enregistrée, le lecteur/spectateur des films de l'écriture peut observer tous les mouvements du curseur, toutes les modifications apportées. Alors qu'un manuscrit donne accès au résultat d'opérations sur la mise en œuvre desquelles les informations sont extrêmement ténues, à partir d'un enregistrement d'écriture, on voit ces opérations se dérouler. Dès lors, la frontière entre ce qui est écrit et ce qui va s'écrire est mouvante, et c'est ce qui induit le caractère problématique de l'ajout : la reconstitution de l'écriture par le logiciel rend visible le fait qu'à tous les moments de la constitution du texte, des mots viennent s'ajouter à d'autres mots déjà écrits.

\subsection{Remplacement versus suppression + ajout}

C'est également à un problème de frontière que nous confronte la notion de remplacement : dans le cas d'une suppression suivie d'un ajout, quand doit-on considérer qu'il s'agit de deux opérations (une suppression simple et un ajout simple), quand faut-il identifier une seule opération (un remplacement) ? Le problème se pose déjà à partir des manuscrits: J. Rey-Debove conclut simplement que c'est »la communauté de projet ou de contenu » qui décide de l'existence ou non d'un remplacement (Rey-Debove 1982, p.114). Ce critère assez flou laisse une grande part à l'initiative personnelle du généticien et il est peu satisfaisant dès lors que l'on n'est pas dans le cas d'un remplacement quasi-synonymique. Le passage par une analyse en constituants syntaxiques paraît plus approprié puisqu'il permet de s'abstraire de considérations sur le projet du lecteur pour s'intéresser aux contraintes de fonctionnement textuel. L'équivalence syntagmatique a en outre l'avantage de se conformer aux critères de l'analyse distributionnelle, d'où sont issues les catégories d'ajout, suppression, remplacement et déplacement.

Les reconstitutions en temps réel posent un problème supplémentaire : celui du remplacement en deux temps. Par exemple, dans la phrase suivante (copie d'un étudiant) :

Le cahier bleu est une œuvre méconnue mais extrêmement intéressante de L. Wittgenstein.

Sont effectuées les modifications suivantes :

- la suppression de l'expansion nominale « de L. Wittgenstein » 
- puis, une heure après, le retour à cet endroit et l'ajout, exactement à la même place, de « de ce philosophe si apprécié aujourd'hui ».

Dans un manuscrit, l'énoncé se présenterait ainsi :

\section{Le cahier bleu est une œuvre méconnue mais extrêmement de ce philosophe si apprécié aujourd 'hui intéressante-de L. Wittgenstein.}

La modification serait classée, sans le moindre doute, comme un remplacement. Avec les données temporelles que livrent les reconstitutions en temps réel, il me semble difficile de tenir cette position : il paraît clair en effet que la suppression de A et l'inscription de B ne s'inscrivent pas dans une opération commune. A était syntaxiquement suppressible, il a été supprimé, et par la suite (même si, au niveau du sens et de la synonymie référentielle, A et B sont en relation symétrique et interchangeables), B a été ajouté. Faut-il pour autant ignorer l'équivalence référentielle et traiter les deux opérations indépendamment l'une de l'autre ? Cette question renvoie au fait que fondamentalement, les opérations d'écriture consistent en des ajouts et des suppressions, et leurs combinaisons éventuelles : remplacer, tout comme déplacer d'ailleurs, c'est supprimer puis ajouter. Il convient donc peut-être de poser l'ajout et la suppression comme les deux opérations fondamentales et de considérer le remplacement (et le déplacement, puisque le même genre de cas peut échoir ${ }^{8}$ ) comme des combinaisons dont la réalisation s'effectue selon des modalités diverses : remplacement enchaîné ou remplacement en deux temps par exemple.

\section{Différentes tentatives de transcrire l'écriture enregistrée}

Nous nous proposons maintenant de traiter des différents essais qui, en une dizaine d'année et de manière très différentes, ont su rendre compte des richesses l'enregistrement de l'écriture.

\subsection{Premier essai : La représentation par reconstitution chronologique}

Du point de vue du lecteur des manuscrits / films de l'écriture, le matériau électronique fait apparaître bien davantage que le papier: toutes les opérations et temps de pause étant notés chronologiquement, il est possible de suivre pas à pas les traces de la constitution d'un texte. À l'inverse des manuscrits où l'espace textuel est donné et l'interprétation chronologique à construire, c'est précisément la chronologie qui organise les reconstitutions de l'écriture sur traitement de texte: à partir des films, on décrit séquentiellement les opérations d'écriture; à partir d'une chronologie explicite, le lecteur doit se représenter l'espace textuel, comme le lecteur de manuscrit se représente la chronologie à partir du matériau qu'il a sous les yeux.

Dans son travail de thèse (Doquet-Lacoste, 2004) et des travaux ultérieurs, Claire Doquet a réutilisé de la manière suivante la classification des opérations d'écriture élaborée à partir des manuscrits :

- $\quad$ ajout $=$ entrée au clavier de tout segment de texte ;

- suppression = effacement d'un segment de texte ;

- déplacement $=$ suppression d'un segment immédiatement suivie de l'ajout du même segment à un autre endroit du texte ;

- remplacement $=$ suppression d'un segment immédiatement suivie, sans pause ni mouvement du curseur, de l'ajout au même endroit d'un segment syntagmatiquement équivalent.

Les pauses d'écriture dont la durée est significative sont également intégrées dans la transcription : on ne peut évidemment pas savoir ce qui se passe pendant ces pauses, mais leur présence est souvent une aide 
précieuse à l'interprétation des phénomènes observés. Dans certaines successions d'opérations, l'absence de pause est elle aussi significative.

Chaque opération est située chronologiquement à la fois dans la durée de l'écriture et au sein des autres opérations effectuées (repère temporel : colonne de gauche dans l'exemple donné plus loin). On dispose ainsi, pour chaque opération, de l'état du texte au moment où elle a eu lieu, ce qui facilite considérablement les hypothèses interprétatives concernant cette opération. Ceci permet d'étudier précisément l'enchaînement des opérations.

Il a paru important de différencier, dans le descriptif de la succession des opérations, celles qui ont lieu à la suite du déjà-écrit de celles qui ont lieu au sein du déjà-écrit. Ces dernières sont présentées dans les reconstitutions sur fond tramé. Les déplacements du curseur sont en outre signalés de manière à restituer le plus précisément possible les traces du parcours du texte. Enfin, pour faciliter la lecture des reconstitutions de l'écriture, sont inclus par tranches de 20 ou 30 minutes des états intermédiaires du texte en cours d'écriture. Ils sont placés en regard de la reconstitution, sur le verso des feuillets, de manière à ne pas entraver la lisibilité des opérations.

Ainsi, dans ce texte (à $10 \mathrm{mn}$ d'écriture) :

Vivre en étant different

La né dernière en 1992 une équipe de canal + a interriouvé des personnes qui ont malheureusement des problèmes : se sont les illéttrés. Etre, que les gens ne penses pas.

les opérations suivantes :

00:10:00 retour après le titre

saut de ligne

création d'un alinéa au premier paragraphe

avancée en fin de texte, après les sauts de lignes

ajout de L'illéttrisme

pause $=00: 01: 08$

remontée en phrase précédente

ajout d'une virgule après Etres.

avancée en fin de texte

ajout de c'est ne savoir ni lire, ni

00:15:00 remontée à la phrase précédente, suppression puis rétablissement de la virgule après Etres

avancée en fin de texte

ajout de écrire

remplacement de $n i$ (lire) par pas parfaitement

remplacement de ni écrire par et mauvaise orthographe.

ajout de Dans la vie quotidienne des illéttrés, il y a beaucoup de problèmes.

remplacement du point par une virgule

00:20:00 ajout de aussi des problèmes dans la vie professionnelle.

pause $=00: 02: 19$

conduisent au texte (à 20 mn d'écriture) :

Vivre en étant different 
La né dernière en 1992 une équipe de canal + a interriouvé des personnes qui ont malheureusement des problèmes : se sont les illéttrés. Etre, que les gens ne penses pas.

L'illéttrisme c'est ne savoir pas parfaitement lire et mauvais orthographe. Dans la vie quotidienne des illéttrés, il y a beaucoup de problèmes, aussi des problèmes dans la vie professionnelle.

L'avantage de cette procédure est l'appui constant sur la spécificité de l'écriture enregistrée : la restitution des variables temporelles (pauses et chronologie) de l'écriture. Son principal défaut est de ne pas faire apparaître clairement le texte lui-même : l'énumération des opérations focalise l'attention sur le niveau micro et, selon la densité des modifications, on perd en lisibilité d'ensemble, chaque opération étant restituée en tant que telle, sans hiérarchisation a priori, sans lien graphique ou spatial avec l'ensemble du texte. Le deuxième problème posé par ce type de transcription est la part de la métalangue : en nommant chaque opération, le transcripteur interprète déjà les données. Nous avons vu plus haut à quel point cette nomination est par ailleurs problématique. Pour tenter de palier l'ambiguïté de la dénomination « ajout », on peut différencier la continuation du texte de sa modification; il a été proposé de nommer la première « inscription » et la seconde "insertion » sans que ces dénominations parviennent à satisfaire l'ensemble des chercheurs.

\subsection{Deuxième essai : La représentation par progression linéaire}

A partir d'un corpus en français langue étrangère (les scripteurs sont finnophones), Leblay (2009 : 164) a proposé la classification suivante :

Tableau 2. Les différents types d'opérations d'écriture

\begin{tabular}{|c|c|c|c|}
\hline \multicolumn{3}{|c|}{ Les différents types d'opérations d'écriture } \\
\hline \multirow{2}{*}{ Opérations d'écriture à la suite du déjà écrit } & \multicolumn{2}{|c|}{ Opérations d'écriture par retour dans le déjà écrit } \\
\hline \multirow{2}{*}{ Opération de type 1 } & Ajout & Ajout & \multirow{2}{*}{ Opérations de type 2} \\
\cline { 2 - 3 } [Variantes d'écriture] & Suppression & Suppression & \multirow{2}{*}{ [Variantes de lecture] } \\
\cline { 2 - 3 } & Remplacement & Remplacement & \\
\cline { 2 - 3 } & Déplacement & Déplacement & \\
\hline
\end{tabular}

Cette classification permet à Leblay de distinguer de manière très économique, dans la nomination, les variantes de lecture des variantes d'écriture, qui correspondent respectivement aux opérations de types 2 et $1 \mathrm{du}$ tableau. Ainsi, il parle de «remplacement $1 »$ ou de «suppression $2{ }^{9}$. Il propose également un nouveau système de transcription des reconstitutions enregistrées. Il s'agit de présenter les données d'écriture au plus près de ce qu'elles seraient dans un manuscrit, avec une reconstitution du texte qui inclut les mots supprimés, qui sont alors biffés (opérations de type 1) et présente en gras les opérations différées, qui ont lieu donc dans une portion de texte déjà écrite, par retour dans ce segment (opérations de type 2). Les déplacements du curseur sont indiqués tantôt avec l'icône de la souris, tantôt avec les icônes des flèches, selon le mode choisi. Chaque modification notée comporte un numéro, ce qui permet de 
reconstituer l'ordre dans lequel ont été effectuées les opérations. Ainsi, dans l'exemple qui suit, par sept fois, le scripteur revient dans le déjà écrit, pour y effectuer principalement des ajouts (segments en gras), des suppressions (segments en gras et raturés), à l'aide des flèches. L'essentiel du travail de révision se voit dans les retours numérotés 5 et 7 :



Cette proposition constitue une réponse à un des écueils de la transcription précédente : le manque de lisibilité du texte. Pour autant, elle ne donne pas toute satisfaction dans la mesure où elle ne donne pas une représentation claire des pauses et ne facilite pas non plus l'appréhension de la chronologie des opérations, même si elle la mentionne.

\subsection{Troisième essai: La visualisation par graphes mathématiques}

A la suite des deux précédentes tentatives, Caporossi et Leblay (2011) ont tenté une étape supplémentaire, en essayant de rendre compte des mêmes phénomènes par une visualisation non linéaire et non verbale, faites à partir de la théorie des graphes mathématiques. Ceux-ci sont une simple conjugaison de cercle et de trait, l'essentiel venant des liens qui les unissent (ou pas), et de l'orientation de ces liens.

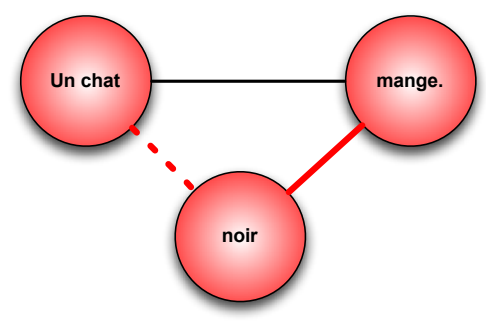

Graphique 3. Construction d'un graphe élémentaire de 3 cellules

Soit un énoncé élémentaire : dans un premier temps, est écrit, d'un seul jet, «Un chat mange. », puis, dans un second temps, avec ou sans pause significative, est inséré l'adjectif noir entre chat et mange. Nous aurons donc un changement de configurations: l'ensemble Un chat mange, d'abord produit en bloc, est ensuite, disjoint par une insertion. Ce qui donne alors une nouvelle configuration de type : Un chat / noir / mange.

Nous pouvons alors représenter ce qui vient de se passer en utilisant un graphe mathématique. Une première lecture, chronologique serait «Un chat / mange / noir», alors qu'une autre lecture, topographique, serait «Un chat / noir / mange.» L'utilisation des graphes permet de conserver simultanément les deux lectures du processus, en isolant les trois éléments que sont un chat, noir et mange. Ces trois éléments sont appelés des cellules de production. 
La première information élémentaire que véhicule toute cellule est donc sa position topographique et chronologique ; à cette information élémentaire (identification du jeu originel des cellules de production), il nous est possible d'en rajouter d'autres ; a) comme celle de savoir combien d'événements sont inclus, à un moment donné, dans une même et seule cellule ; ou bien encore b) comme celle de savoir si telle ou telle cellule résistera, ou non, aux reformulations de mise en texte pour apparaître dans le texte (final).

Mais il est possible de rajouter d'autres informations dans le mode de visualisation : la première concerne la taille des cellules qui notera le nombre d'événements produits au clavier ou à la souris : plus une cellule est grosse, plus elle comprend d'événements. La seconde information a trait au jeu de couleurs utilisées (deux ou trois) : les cellules, toutes colorées, peuvent anticiper le texte (final) en distinguant celles qui vont disparaître de celles qui vont subsister. Ainsi, dans l'exemple suivant (graphique 4), nous proposons un jeu de trois couleurs : les cellules jaunes et rouges sont des cellules d'ajout, tandis que les cellules bleues sont des cellules de suppression.

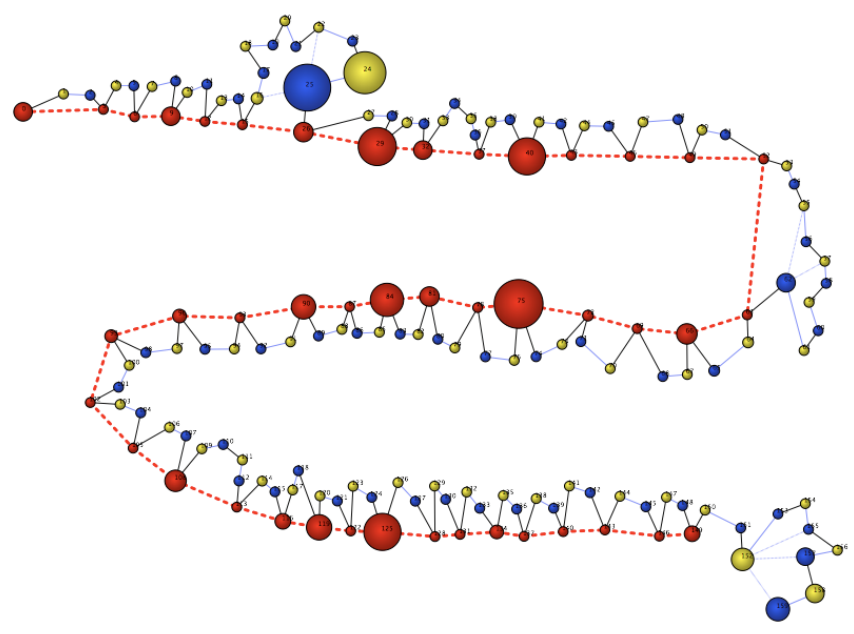

Graphique 4. Graphe de 15 minutes d'enregistrement

Il est possible d'aller bien plus loin dans la notation des données offertes par les enregistrements, grâce aux liens. Les cellules entretiennent avec d'autres cellules des liens contingents : l'un chronologique, l'autre topographique. Ainsi, un lien chronologique, marquant la succession, entre deux cellules qui ne seront pas supprimées (donc en rouge) sera noté d'un trait gras (le fameux fil rouge). Les lignes en trait continu représentant les liens chronologiques, il est donc possible de suivre le processus de manière chronologique en suivant uniquement les lignes continues tandis qu'une lecture topographique se fera par les lignes rouges. Les cellules de suppression portent bien sûr sur une portion de texte qui avait été ajoutée précédemment. Une ligne bleue relie la cellule de suppression à la cellule d'ajout qui lui correspond et cette dernière sera représentée en jaune.

Nous avons donc cherché à représenter comment, du point de vue de la production, une unité n'est pas $a$ priori stable. Rien ne peut prédire, au moment où celle-ci est énoncée, la position qu'elle occupera au sein du texte final. Cette unité y sera-t-elle encore présente? Ou bien aura-t-elle complètement disparue du texte ? Ou bien encore occupera-t-elle cette même place ? Tout est possible dans cet univers de production où, malgré tous les efforts de planification anticipée, aucune unité ne peut être tenue à l'écart des effets de production. Cette unité changeante, hautement fonctionnelle, a reçu le nom de cellule. Celle-ci désigne une unité vivante, en plein devenir, au sein d'un ensemble qui est en train de prendre corps, de s'organiser. 


\subsection{Représentation, visualisation \& nouveau métalangage}

A considérer ces trois essais, dans leurs évolutions successives (le premier autorisant le deuxième qui autorise le troisième), il serait légitime de s'interroger sur le retour, le feedback, proposée aux scripteurs. L'apport technologique doit pouvoir se faire sentir autant dans le choix de nouveaux supports d'écriture que dans celui de nouveaux outils pour représenter ce qui s'est passé durant l'écriture. Il n'est plus suffisant de proposer un mode d'écriture numérique enregistrée sans proposer des outils de feed-back adaptés. N'y aurait-il pas, trop souvent encore, un décalage entre des nouveaux outils d'écriture et les outils d'évaluation de ces écritures? Nous proposons de procéder en 3 temps :

Tableau 3. Vers un nouveau métalangage?

\begin{tabular}{|c|c|c|c|}
\hline Papier versus écran & A. Logiciels & B. Evaluation verbalisée & $\begin{array}{c}\text { C. Evaluation non } \\
\text { verbalisée }\end{array}$ \\
\hline 1. Ecriture sur papier & - & - Crayon & $\begin{array}{c}\text { - Marques } \\
\text { (soulignage et ratures) }\end{array}$ \\
\hline \multirow{2}{*}{ 2. Ecritures sur écran } & $\begin{array}{c}\text { Traitement de texte } \\
\text { [Word, Pages, }]\end{array}$ & $\begin{array}{c}\text {-Fonctions Commentaire, } \\
\text { Révision (onglet spécial } \\
\text { dans Word) }\end{array}$ & - Utilisation des couleurs \\
\cline { 2 - 4 } & $\begin{array}{c}\text { Logiciel } \\
\text { d'enregistrement } \\
\text { [Genèse du texte, } \\
\text { ScriptLog, InPutLog, } \\
\text { TransLog...] }\end{array}$ & $\begin{array}{c}\text { - Possible sous la forme } \\
\text { d'entretiens }\end{array}$ & $\begin{array}{c}\text { Graphes visualisant la } \\
\text { dynamique scripturale, } \\
\text { utilisation des couleurs et } \\
\text { des formes }\end{array}$ \\
\hline
\end{tabular}

Deux modes déterminent la dynamique d'écriture, l'un sur papier (1), l'autre sur écran (2). Loin de s'exclure mutuellement, ils se complètent, l'un apportant des modalités que l'autre ne pouvait connaître (utilisations de logiciels ou non). C'est précisément dans le choix déterminant des logiciels d'aide à l'écriture (A) qu'il convient de s'arrêter: deux types de logiciels sont disponibles. Les logiciels de traitement de texte, comme Word ou Pages doivent être distingués de ceux qui permettent l'enregistrement de l'écriture (Genèse du texte, ScriptLog, InPutLog, TransLog...).

Nous proposons, dans ce travail, non seulement un rappel de ces deux supports d'écriture (papier versus écran), mais aussi des deux principaux types de logiciels. Pour simplifier à l'extrême, il serait possible de dire que les logiciels de traitement de texte sont des logiciels dédiés à l'écrit (traiter du texte), tandis que les logiciels d'enregistrement sont dédiés à l'écriture (enregistrer de l'écriture).

Il existe, comme on peut s'y attendre, des pratiques évaluatives bien différentes pour rendre compte de ces deux modes d'appréhension de l'écrit ou de l'écriture. On peut en rendre compte verbalement (B), ou non verbalement (C). Tant les pratiques développées avec l'usage du papier et du crayon que celles avec un écran et un clavier permettent des évaluations verbalisées ; les annotations écrites en marge (sur papier) et les commentaires, ajouts, suppressions insérés avec l'aide de fonctions comme Commentaire ou Révision (sur écran) permettent de donner un feed-back utile et nécessaire au scripteur; de même, il est possible de discuter directement avec le scripteur pour commenter de vive voix ce qu'il a écrit. Mais il est tout aussi possible d'évaluer sans verbalisation explicite: ainsi un surlignage sur papier ou bien sur écran permet 
d'attirer l'attention du scripteur sur certains points. C'est en poursuivant cette direction que nous proposons un mode d'évaluation par graphe: celui-ci est un mode numérique, produit à partir de logiciels d'enregistrement. L'idée est bien alors de mettre le scripteur dans une situation de regarder son écriture comme s'il s'agissait de l'écriture d'un(e) autre, en présence du dynamisme de l'écriture, dans une situation d'observateur.

En fin de compte, n'est-il pas légitime de se demander si les représentations et les visualisations des dynamiques d'écriture ne sont pas de nouveaux métalangages? Ne représentent-ils pas une autre manière de faire retour sur le processus d'écriture ? Ne sont-ils le retour attendu face aux défis mis en place par les écritures numériques. Il semble légitime que l'écriture sur écran donne lieu à des évaluations d'un autre ordre, susceptible de rendre compte du changement de technologie, c'est-à-dire à des évaluations réalisées sur écran et pas sytématiquement verbalisées.

Les deux représentations (celle par reconstitution chronologique et celle par progression linéaire) et la visualisation par graphes offrent aux scripteurs eux-mêmes la possibilité de porter un regard distancié sur la manière dont ils viennent d'écrire, à l'écart des annotations en marge et des commentaires faits de vive voix, signes habituels d'un regard extérieur.

\section{Pour finir}

La génétique textuelle, à partir de descriptions et d'interprétations (transcriptions) faites pour appréhender l'espace des manuscrits sur papier, a su donc mettre en place une description du temps comme succession. En accueillant et en utilisant de nouveaux outils, elle a réussi à mettre en place une description du temps comme durée ; d'où une triple dimension spatio-temporelle génétique : tout d'abord, l'espace, ensuite le temps, décrit dans sa succession, enfin le temps, décrit dans sa durée. Le passage d'une technologie du papier à une technologie de l'écran est déterminant.

Sans pouvoir préjuger du remplacement, partiel ou total, d'une écriture (sur papier) par une autre (sur écran), il reste que, pour l'instant, ces deux modes d'écritures co-existent. Quoique chacun de ces modes d'écriture corresponde à des pratiques et des usages différents, ils n'en reflètent pas moins des pratiques unifiées par des invariants. Nous connaissons bien maintenant les pratiques (et les limites) induites par l'usage du papier et du crayon. Mais nous ne pouvons que nous interroger sur l'usage de l'écran, en particulier en situation de production scolaire et universitaire. L'usage de la technologie de l'écran induit non seulement de nouveaux modes d'écriture, mais aussi de nouveaux modes d'évaluation de ces écritures.

Les représentations et visualisations, véritables nouveaux métalangages, sont de vraies réformes dans la lecture de la temporalité au sein de l'écriture : elles concernent autant la description que l'analyse des phénomènes génétiques et linguistiques impliqués.

\section{Références bibliographiques}

Bellemin-Noël, X. (1982). Avant-texte et lecture pyschanalytique. In L. Hay et P. Nagy Avant-texte, texte, après-texte, Actes du Colloque international de textologie à Mátrafüred (Hongrie), 13-16 octobre 1978. Akadémiai kiadó, 161165.

Caporossi, G. et Leblay, C. (2011). Online Writing Data Representation: a Graph Theory Approach. In J. Gama, E. Bradley \& J. Hollmen (Ed.), Advances in Intelligent Data Analysis X-10th International Symposium, IDA 2011, Porto, Lecture Note In Computer Science 7014, 80-89. 
Chesnet D., Alamargot D. (2005) Analyse en temps réel des activités oculaires et grapho-motrices du scripteur : intérêt du dispositif « Eye and Pen ». In: L'année psychologique, vol. 105, n³. pp. 477-520.

Chesnet D., Guillabert F., Esperet E. (1994) G-STUDIO : un logiciel pour l'étude en temps réel des paramètres temporels de la production écrite. In: L'année psychologique, vol. 94, n². pp. 283-293.

Debray-Genette, R. (1982), « Génétique et théories littéraires », in Hay, L. Le Texte et l'avant-texte, p.167-170

Doquet, C. (1995) Le Temps d'écrire : stratégies d'écriture et chronologie des événements dans des processus d'écriture d'élèves de CM2. Repères, $\mathrm{n}^{\circ} 11.59-72$.

Doquet-Lacoste, C. (2004). Indices et traces de l'activité métadiscursive des scripteurs. Aspects de la réécriture. Le français d'aujourd'hui 144, 33-41.

Fenoglio I., « Genèse du geste linguistique : une complexité heuristique », GENESIS, manuscrits, recherche, invention, $\mathrm{n}^{\circ} 35$, « Le geste linguistique, textes réunis et présentés par Irène Fenoglio », 2012, p.13-40

Fenoglio, I. (2009). Les notes de travail d'Emile Benveniste : où la pensée théorique naît via son énonciation, Langage \& Société 127, 23-49.

Fenoglio, I. (2007). Ed. L'écriture et le souci de la langue, Louvain-la-Neuve : Academia-Bruylant.

Fenoglio I. (2003) Graphie manquée, lapsus écrit : un acte d'énonciation attesté, Langage et Société 103, 57-77.

Foulin, J.-N. (1988) Etude en temps réel de la production eEcrite chez des enfants de sept et huit ans. European Journal of Psychology of Education, Volume 3, Issue 4, pp 461-475

Foulin, J.-N., Chanquoy, L., Fayol, M. (1989) «Approche en temps réel de la production des connecteurs et de la ponctuation : vers un modèle procédural de la composition écrite », Langue Française, n81, p.5-20.

Grésillon, A. (1994). Éléments de critique génétique: lire les manuscrits modernes. Paris : PUF.

Grésillon, A. (1993) «Méthodes de lecture», in Hay, L. (ed) Les Manuscrits des Ecrivains, Paris, Hachette-CNRS. p.138-161.

Grésillon, A. (1990). Les manuscrits littéraire : le texte dans tous ses états. In A. Gresillon, J.-L. Lebrave \& C. Viollet, Proust à la lettre. Les intermittences de l'écriture. Tusson : Du Lérot, 15-42.

Grésillon, A. (1989). Fonctions du langage et genèse du texte. In L. Hay (éd.), La naissance du texte. Paris : José Corti, 177-192.

Hay, L. (2009). Réflexions sur la discipline. In O. Anokhina et S. Pétillon, Critique génétique. Concepts, méthodes, outils, Actes de l'école thématique de l'ITEM. Critique génétique : Manuscrits, écriture, invention, IMEC 20-24 septembre 2004, IMEC éditeur, 21-31.

Hay, L. (2002). La littérature des écrivains. Questions de critique génétique. Paris : Corti.

Leblay, C. (2009). La question du déjà écrit dans le processus d'écriture observé en temps réel. Une contribution de la génétique à la didactique. In I. Fenoglio \& J.-M. Adam (éd.) Génétique de la production écrite et linguistique, Modèles linguistiques, Tome XXX, volume 59, 153-176.

Leblay et Caporossi. (2014, à paraître). Ed. Le temps de l'écriture. Enregistrements et représentations. Xx : AcademiaL'Harmattan.

Lebrave J.-L., «Manuscrits de travail et linguistique de la production écrite », Modèles linguistiques, 30, n59, "Génétique de la production écrite et linguistique / volume coordonné par Irène Fenoglio et Jean-Michel Adam », 2009, p.13-21 
Rey-Debove, J. (1982), «Pour une lecture de la rature », Fuchs, C., Grésillon, A., Lebrave, J.-L. et al., La Genèse du texte: les modèles linguistiques, p.21-72.

1 Cette équipe historique se nomme aujourd'hui « Manuscrits, linguistique, cognition ».

2 Le terme de substitution, employé par les généticiens au moins au début de leurs travaux, pose un problème de confusion avec l'une des substitutions, le remplacement (d'ailleurs lui-même nommé, parfois, « substitution »). C'est la raison pour laquelle nous lui préfèrerons dorénavant le terme d'opération, qui a en outre l'avantage de mettre l'accent sur le processus plutôt que sur son résultat.

3 "Les diverses étapes de retours sur du déjà écrit ne s'inscrivent pas sur une ligne droite qui conduirait sans faille vers l'idéal du texte définitif. La vision téléologique d'une avancée de l'écriture vers l'achèvement, c'est-à-dire vers la perfection, est contredite par les manuscrits eux-mêmes." (Grésillon, 1990, p. 32)

4 "On reconnaît pour clos un texte où convergent la finitude, la finition et la finalité. La finitude est aléatoire, elle peut relever d'un arbitraire motivé après coup. La finition est le sentiment d'achèvement. Rien ne vous dit si ce sentiment vient de celui de perfection ou de celui de lassitude. La finition est souvent l'effet d'une saturation structurale qui bloque en cours de route tout ou partie de l'œuvre. Quant à la finalité, elle est certes importante, mais les écrivains ont cent fois avoué que leur œuvre avait trahi leur projet. De sorte que la clôture d'un texte est plutôt une hypothèse d'école qu'une évidence scientifique." (Debray-Genette, 1982, p. 167)

5 Cf. le titre d'un numéro 103 de la revue Langage et Société : Ecriture en acte et genèse du texte (Fenoglio 2003).

6 «En abordant l'écriture, la critique rencontre inéluctablement une instance écrivante, tendue entre le vécu et la forme et qui parcourt cet espace de tensions par le sillage de la plume.» (Hay, 2002, p. 55)

$7 \mathrm{Il}$ arrive que les écrivains qui utilisent le traitement de texte de dessinent, mais cela se fait alors sur une sortie papier, comme l'a montré I. Fenoglio sur des manuscrits de Pascal Quignard ("Pascal Quignard. Dessins, images comme lecture "for-intérieure" de l'oeuvre à venir" colloque IMEC, Les dessin d'écrivains, 18-19 fevrier 2008) (cf. Fenoglio 2007 et Fenoglio 2009).

8 Soit par exemple l'énoncé suivant: « Nous partîmes à l'aube» qui devient «A l'aube nous partîmes ». Si l'on compare les deux énoncés, on observe qu'il y a eu déplacement du GP, mais les choses peuvent très bien s'être déroulées ainsi : (1) inscription de « Nous partîmes à l'aube », (2) suppression de «à l'aube », (3) une heure plus tard, ajout de «A l'aube» avant «nous partîmes ». Sur le plan strictement procédural, il est alors difficile de parler de déplacement, alors que sur le plan des équivalences syntagmatiques le déplacement (GNS + GV + GP > GP + GNS + $\mathrm{GV})$ est effectif.

9 Leblay introduit même la notion d'opération de type 3, réservée aux opérations de modification d'un segment déjà écrit qui avait fait lui-même l'objet d'une écriture au sein du déjà écrit (Leblay, 2009 : 169). 